\title{
Применение свёрточных нейронных сетей для компенсации нелинейных эффектов в волоконно-оптических линиях связи со спектральным уплотнением каналов
}

\author{
$\underline{\text { О.С. Сидельников }}^{1, *}$, А.А. Редюк ${ }^{1,2}$, М.П. Федорук ${ }^{1,2}$, С.К. Турицын ${ }^{1,3}$ \\ ${ }^{1}$ Новосибирский государственный университет \\ ${ }^{2}$ Институт вычислительных технологий СО РАН \\ ${ }^{3}$ Институт фотонных технологий, университет Астона \\ *E-mail: o.sidelnikov@g.nsu.ru
}

DOI: 10.31868/RFL2020.122-123

Нелинейность оптического волокна является основным ограничивающим фактором для дальнейшего увеличения пропускной способности современных систем передачи информации. Эксплуатация линий связи со спектральным уплотнением каналов (Wavelength-division multiplexing - WDM) предполагает увеличение общей мощности сигнала в волокне, что приводит к растущему воздействию нелинейных эффектов передачи. Метод обратного распространения сигнала (Digital backpropagation - DBP) [1] на сегодняшний день является одним из наиболее эффективных схем компенсации нелинейных искажений, однако он обладает высокой вычислительной сложностью. Методы машинного обучения и нейронные сети (HC), в частности, в настоящее время стали активно применяться в области волоконнооптических линий связи благодаря тому, что они обеспечивают высокую точность классификации принятых символов при небольшой сложности [2]. В данной работе мы предлагаем схему компенсации нелинейных эффектов в линиях связи со спектральным уплотнением каналов, основанную на свёрточных нейронных сетях и имитирующую метод обратного распространения сигнала.

В работе рассматривается система передачи данных, состоящая из передатчика, 40 пролетов стандартного одномодового волокна по 80 км каждый, эрбиевых оптических усилителей после каждого пролета и приемника. На передатчике формируются 16-QАМ сигналы с символьной скоростью $\mathrm{R}_{\mathrm{s}}=64$ Гбод. Для придания формы импульсам используется фильтр с характеристикой типа корень из приподнятого косинуса с коэффициентом сглаживания 0.1. Исследуется система передачи данных с 11 частотными каналам с межканальным интервалом равным 75 ГГц. После распространения по каналу принятый сигнала проходит через согласованный фильтр, и после понижения частоты дискретизации выполняется компенсация нелинейных эффектов с использованием предложенной схемы. Далее выполняется демодуляция сигнала и вычисления коэффициента битовых ошибок (Bit error rate - BER).

Архитектура предложенной в работе глубокой свёрточной нейронной сети изображена на Рис. 1(a). Она моделирует метод обратного распространения сигнала, основанного на связанных нелинейных уравнениях Шредингера, и состоит из чередующихся слоев, соответствующих компенсации дисперсионных и нелинейных эффектов. Линейные слои такой нейронной сети представляют собой свёрточные фильтры, которые позволяют эффективно компенсировать хроматическую дисперсию, обладая при этом невысокой вычислительной сложностью. Нелинейная функция активации соответствует нелинейному шагу DBP с использованием расширенного метода Фурье расщепления по физическим процессам [3]. На вход свёрточной нейронной сети подаются принятые сигналы с одним отсчетом на символ, причем 
рассматриваемые частотные каналы и поляризационные компоненты обрабатываются параллельно. Нами была реализована комплексная архитектура НС с использованием библиотеки MXNet. B качестве функции ошибок использовалась среднеквадратичная ошибка между переданными 16-QАМ символами, и символами, полученными на выходе из нейронной сети. В работе используется глубокая свёрточная нейронная сеть, состоящая из 40 слоев, что соответствует числу пролетов в рассматриваемой линии связи.
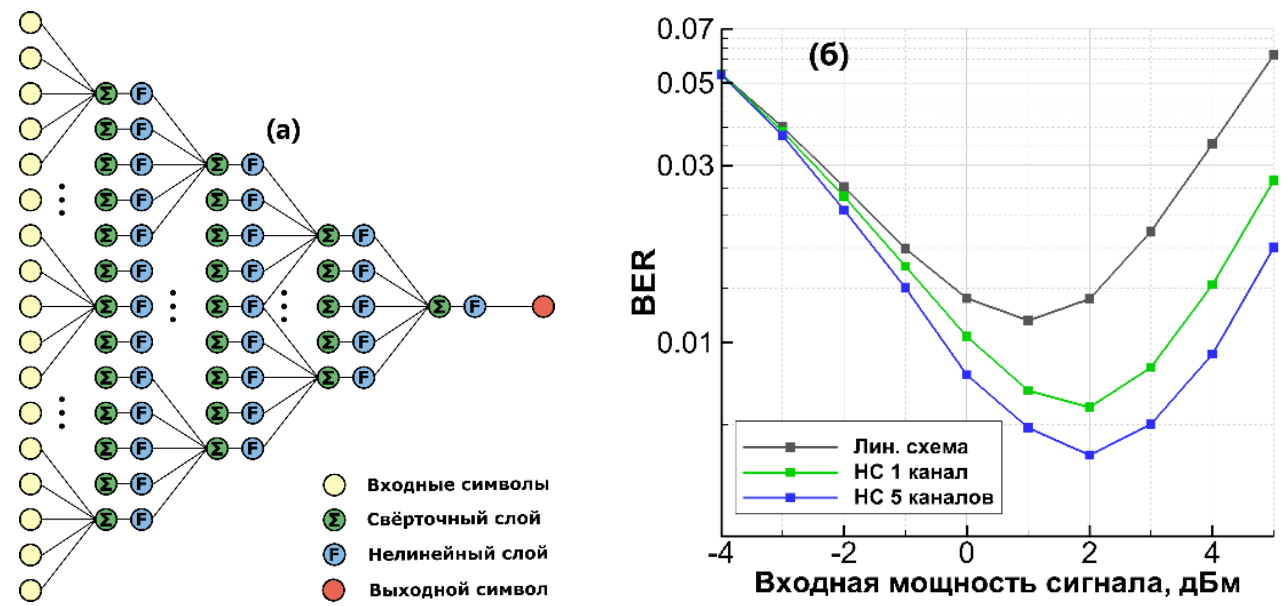

Рис. 1. (а) Архитектура рассматриваемой нейронной сети и (б) зависимость BER от начальной мощности сигнала.

Для оценки эффективности предложенной схемы мы сравнили линейную схему компенсации, которая только восстанавливает фазу принятого сигнала, и метод основанный на глубоких свёрточных сетях, который одновременно обрабатывает один и пять частотных каналов. На Рис. 1(б) представлена зависимость коэффициента битовых ошибок от начальной мощности сигнала для различных схем компенсации нелинейности. Как мы можем видеть, за счет эффективной компенсации нелинейных эффектов фазовой самомодуляции свёрточная нейронная сеть позволяет снизить BER при оптимальной мощности на $42 \%$ в сравнении с линейной схемой компенсации. В случае, когда предложенная схема обрабатывает 5 спектральных каналов одновременно, она дополнительно может компенсировать эффекты фазовой кроссмодуляции. Такая схема демонстрирует коэффициент битовых ошибок на 58\% меньше, чем линейная схема, и на $27 \%$ в сравнении с «одноканальной» нейронной сетью. Следует также отметить, что обе глубокие свёрточные НС имеют одинаковую вычислительную сложность.

Исследование выполнено при поддержке Российского научного фонда (грант № 17-72-30006).

\section{Литература}

[1] E. Ip, J.M. Kahn, J. Lightwave Technology 26, 3416-3425 (2008)

[2] O.S. Sidelnikov, A.A. Redyuk, S.Sygletos, Opt. Express. 26, 32765-32776 (2018).

[3] M. Secondini, D. Marsella, E. Forestieri, Proc. ECOC, We.3.3.5. (2014). 